\title{
The social determinants of pandemic impact: an opportunity to rethink what we mean by "public health spending"
}

\section{Les déterminants sociaux de l'impact des pandémies : une possibilité de repenser ce que nous voulons dire par les « dépenses de santé publique »}

\author{
Lindsay McLaren ${ }^{1} \cdot$ Daniel J. Dutton ${ }^{2}$ \\ Published online: 11 August 2020 \\ (C) The Canadian Public Health Association 2020
}

An important paper recently published in the Canadian Journal of Public Health showed a decline in financial investment in public health in the province of Quebec. Based on an analysis of health spending data in Quebec from 2004-2005 to 2017-2018, Fiset-Laniel et al. (2020) identified that public health budgets in that province "suffered from disproportionately low investments and abrupt cuts", which were not seen in other areas of health spending.

Funding cuts, or inadequate increases in funding, to public health system activities in Quebec and elsewhere (e.g., Elliott 2020) are understandably upsetting and frustrating to many in the field, perhaps particularly to those on the front line who see the consequences of failing to invest in prevention and protection.

Those consequences are, as they have always been, magnified by a pandemic (Rutty and Sullivan 2010), with COVID19 being just the latest in a historical string of examples where public health infrastructure is mobilized reactively, only to be scaled back or dissolved once the outbreak has passed.

This story, however, of inadequate investment in the budget line-item "public health" is narrow and it distracts from policymaking efforts towards population well-being and health equity.

In a definition familiar to many of us, public health is "the art and science of preventing disease, prolonging life and promoting health through the organized efforts of society" (Last

Lindsay McLaren

lmclaren@ucalgary.ca

1 University of Calgary, Calgary, AB, Canada

2 Dalhousie University, Halifax, NS, Canada
2001). In other words, public health - by definition - embraces the social determinants of health (CPHA 2017) and recognizes, as per a health-in-all-policies approach (Kershaw 2018; Dutton et al. 2018), that organized societal efforts to prevent illness and promote well-being, in an equitable manner, go beyond the health care sector, within which formal public health activities are situated.

Definitions of public health spending, which are currently being mobilized in the pandemic to advocate for greater investment, are not aligned with this definition of public health. This misalignment constitutes an institutionalized barrier to real improvements to population health, well-being, and health equity (i.e., the goals of public health), in pandemic circumstances or otherwise.

The COVID-19 pandemic presents an opportunity to rethink what we mean by "public health spending". As with all health problems, the pandemic reminds us that health impacts are experienced inequitably, reflecting "the conditions in which people are born, grow, live, work and age" (CSDH 2008). That is, as clearly (if not uniquely) demonstrated by the COVID-19 pandemic, one cannot separate formal public health system activities from the social determinants of health. Yet, that is exactly what our best available spending data, and analyses of public health spending, do. That is, they operationalize public health spending in a way that explicitly sets aside the social determinants of health, or that treats them as complementary rather than core.

In the excellent work of the Canadian Institute for Health Information (CIHI), for example, public health spending is defined as including government expenditures on "disease prevention and health promotion activities, community mental health and addictions services, and occupational health services to promote and enhance health and safety in the 
workplace" (CIHI 2019). This definition does not include spending in other areas of government (i.e., spending on the social determinants of health) which produce the conditions that determine how pandemics are experienced.

An expanded definition of public health spending that includes spending on the social determinants of health would align much better with public health as defined above, and would likely illustrate that "public health spending" has decreased, or failed to increase, to an even greater degree than is captured in analyses that use a narrow definition, such as that by Fiset-Laniel et al. (2020). This is because, while health care spending in Canada (of which formal public health system activities constitute a tiny proportion) has increased steadily over the past several decades, social spending - much lower to begin with - has flatlined or decreased (Dutton et al. 2018).

"There is a difference between the broad concept of public health and formal public health services" (Rachlis 2016). This difference is deceptively significant: the fact that there are two versions of public health is getting in the way of real advances towards population health, well-being, and health equity. How we define "public health spending" is not innocuous: it institutionalizes the two versions, including the dominance of the narrow one.

If public health is truly about preventing illness and promoting health and well-being for all, via organized societal efforts, should we not entertain the idea that other forms of government spending, outside of the health care sector, be part of our definitions of public health spending? Such a rethought definition would position a united public health community (Lucyk and McLaren 2017) to advocate for better investment in the health and health equity of the population (i.e., the goals of public health), regardless of which ministry is responsible.

The commentary by Fiset-Laniel et al. (2020) states, "More attention to, and adequate investment in, both public health and social determinants of health are needed to protect population health during epidemics and beyond". As long as we continue to talk about "both public health and the social determinants of health" (italics added) as though they are separate, we will not make real advances towards population health, well-being, and health equity.

Lindsay McLaren, Senior Editor, CJPH; Professor, Community Health Sciences, University of Calgary

Daniel Dutton, Assistant Professor, Community Health and Epidemiology, Dalhousie University

\section{Éditorial}

Un important article paru récemment dans la Revue canadienne de santé publique fait état d'une baisse des investissements financiers en santé publique au Québec. D'après leur analyse des données sur les dépenses de santé au Québec entre 2004-2005 et 2017-2018, Fiset-Laniel et collègues (Fiset-Laniel et al. 2020) ont déterminé que les budgets de santé publique dans la province ont souffert d'investissements démesurément faibles et de compressions brutales qui n'ont pas affecté d'autres domaines des dépenses de santé.

Les compressions, ou les hausses insuffisantes, des budgets des activités du système de santé publique au Québec et ailleurs (voir p. ex. Elliott 2020) sont naturellement affligeantes et frustrantes pour de nombreux professionnels $\mathrm{du}$ domaine, surtout peut-être pour les intervenants de première ligne qui voient les conséquences de l'omission d'investir dans la prévention et la protection.

Ces conséquences sont, comme elles l'ont toujours été, amplifiées par les pandémies (Rutty and Sullivan 2010), la COVID-19 étant simplement la dernière en date d'une liste historique d'exemples où l'on a réagi en mobilisant les infrastructures de santé publique pour ensuite les réduire ou les dissoudre une fois l'éclosion passée.

Cette façon de parler des investissements insuffisants dans l'objet de dépense « santé publique » est cependant étroite et détourne l'attention des efforts à déployer pour élaborer des politiques de bien-être et d'équité en santé de la population.

Selon une définition bien connue, la santé publique est « la science et l'art de prévenir les maladies, de prolonger la vie et de promouvoir la santé au moyen d'une action sociétale organisée » (Last 2001). Autrement dit, la santé publique par définition - adhère aux déterminants sociaux de la santé (CPHA 2017) et reconnait, comme le veut l'approche de la santé dans toutes les politiques (Kershaw 2018; Dutton et al. 2018), qu'une action sociétale organisée pour prévenir les maladies et promouvoir le bien-être, de façon équitable, dépasse le secteur des soins de santé dans lequel s'inscrivent officiellement les activités de santé publique.

Les définitions des dépenses de santé publique invoquées durant la pandémie en cours pour promulguer des investissements plus importants ne sont pas conformes à cette définition de la santé publique. Ce décalage constitue un obstacle institutionnalisé à l'amélioration réelle de la santé, du bien-être et de l'équité en santé de la population (les objectifs de la santé publique), que ce soit en situation de pandémie ou non.

La pandémie de COVID-19 offre la possibilité de repenser ce que nous voulons dire par les " dépenses de santé publique ». Comme tout autre problème de santé, la pandémie nous rappelle que les effets sanitaires sont éprouvés de façon inéquitable et qu'ils reflètent « les circonstances dans lesquelles les individus naissent, grandissent, vivent, travaillent et vieillissent » (CSDH 2008). Autrement dit, comme le démontre clairement (mais pas uniquement) la pandémie de COVID-19, on ne peut pas séparer les activités du système de santé publique officiel des déterminants sociaux de la santé. Pourtant, c'est exactement ce que font les meilleures données et analyses disponibles sur les dépenses de santé publique. Elles opérationnalisent ces dépenses 
en mettant explicitement de côté les déterminants sociaux de la santé ou en les traitant comme s'ils étaient complémentaires et non centraux.

Dans un excellent document de l'Institut canadien d'information sur la santé (ICIS), par exemple, les dépenses de santé publique sont définies comme incluant les dépenses gouvernementales « liées aux activités de prévention des maladies et de promotion de la santé, aux services communautaires en santé mentale et en toxicomanie, et aux services de santé au travail visant à promouvoir et améliorer la santé et la sécurité en milieu de travail » (CIHI 2019). Cette définition n'inclut pas les dépenses du gouvernement dans d'autres domaines (c.-à-d. les dépenses consacrées aux déterminants sociaux de la santé), bien qu'elles produisent les conditions qui déterminent l'expérience vécue des pandémies.

Une définition élargie des dépenses de santé publique, incluant les dépenses consacrées aux déterminants sociaux de la santé, cadrerait mieux avec la santé publique telle que définie plus haut et montrerait probablement que les « dépenses de santé publique » ont diminué, ou n'ont pas augmenté, dans une mesure encore plus grande que ce qu'indiquent les analyses qui utilisent une définition plus étroite, comme celle de Fiset-Laniel et collègues (Fiset-Laniel et al. 2020). En effet, même si les dépenses de soins de santé au Canada (dont les activités du système de santé publique officiel représentent une proportion minuscule) augmentent de façon soutenue depuis plusieurs décennies, les dépenses sociales - beaucoup moins importantes au départ - plafonnent ou diminuent (Dutton et al. 2018).

« Il y a une différence entre la notion générale de santé publique et les services de santé publique officiels » (Rachlis 2016, traduction libre). Cette différence est faussement significative : la coexistence de deux versions de la santé publique fait obstacle à de réelles avancées dans la voie de la santé, du bien-être et de l'équité en santé de la population. La façon dont nous définissons les « dépenses de santé publique » n'est pas anodine : elle institutionnalise les deux versions, et la domination de la plus étroite des deux.

Si la santé publique consiste vraiment à prévenir les maladies et à promouvoir la santé et le bien-être de tous, au moyen d'une action sociétale organisée, ne devrions-nous pas caresser l'idée que d'autres formes de dépenses publiques, hors du secteur des soins de santé, entrent dans nos définitions des dépenses de santé publique? Avec une définition ainsi repensée, la communauté de la santé publique unie (Lucyk and McLaren 2017) serait en mesure de promulguer de meilleurs investissements dans la santé et l'équité en santé de la population (c.-à-d. dans les objectifs de santé publique), quel que soit le ministère responsable.

Dans leur commentaire, Fiset-Laniel et collègues (FisetLaniel et al. 2020) déclarent qu'il faut accorder plus d'attention et consacrer plus d'investissements à la fois à la santé publique et aux déterminants sociaux de la santé (nos italiques) pour protéger la santé de la population pendant les épidémies et au-delà. Tant que nous continuerons de parler comme si la santé publique et les déterminants sociaux de la santé étaient distincts, nous ne ferons pas de progrès réels vers la santé, le bien-être et l'équité en santé de la population.

Lindsay McLaren, Rédactrice scientifique adjointe, RCSP; professeure, Community Health Sciences, University of Calgary

Daniel Dutton, professeur assistant, Community Health and Epidemiology, Dalhousie University

\section{References/Références bibliographiques}

Canadian Institute for Health Information (CIHI) (2019). Technical note: Public health and community mental health and addictions services in NHEX. https://www.cihi.ca/sites/default/files/document/nhexph-cmh-technical-note-2019-en-web.pdf. Accessed 28 Jul 2020.

Canadian Public Health Association (CPHA) (2017). CPHA Working Paper: Public health - a conceptual framework. Ottawa: CPHA https://www.cpha.ca/sites/default/files/uploads/policy/phframework/phcf_e.pdf. Accessed 28 Jul 2020.

Commission on Social Determinants of Health (CSDH) (2008). Closing the gap in a generation: health equity through action on the social determinants of health. Geneva: World Health Organization. https:// www.who.int/social_determinants/thecommission/finalreport/en/. Accessed 28 Jul 2020.

Dutton, D. J., Forest, P.-G., Kneebone, R. D., \& Zwicker, J. D. (2018). Effect of provincial spending on social services and health care on health outcomes in Canada: an observational longitudinal study. CMAJ, 190(3), E66-E71.

Elliott, M. Toronto's COVID-19 response shows the value of a wellfunded public health department. So why the push to cut funding? The Star, March 16, 2020. https://www.thestar.com/opinion/ contributors/2020/03/16/torontos-covid-19-response-shows-thevalue-of-a-well-funded-public-health-department-so-why-the-pushto-cut-funding.html. Accessed 28 Jul 2020.

Fiset-Laniel, J., Guyon, A., Perrault, R., \& Strumpf, E. C. (2020). Public health investments: neglect or willful omission? Historical trends in Quebec and implications for Canada [commentary]. Canadian Journal of Public Health, 111(3), 383-388.

Kershaw, P. (2018). The need for health in all policies in Canada. CMAJ, 190(3), E64-E65.

Last, J. (ed) (2001). A dictionary of epidemiology, 4th edn. New York: Oxford University Press.

Lucyk, K., \& McLaren, L. (2017). Commentary - Is the future of "population/public health" in Canada united or divided? Reflections from within the field. Health Promotion and Chronic Disease Prevention in Canada, 37(7), 223-228.

Rachlis, M. (2016). Canada's Public Health System. CPHA webinar, October 6. https://www.cpha.ca/canadas-public-health-system. Accessed 28 Jul 2020.

Rutty, C., Sullivan, S.C. (2010) This is public health: a Canadian history. Ottawa: CPHA. https://www.cpha.ca/sites/default/files/assets/ history/book/history-book-print_all_e.pdf. Accessed 28 Jul 2020.

Publisher's note Springer Nature remains neutral with regard to jurisdictional claims in published maps and institutional affiliations. 